\title{
PANCAKE COLLAPSE AND STRUCTURE FORMATION FROM CDM DENSITY FLUCTUATIONS
}

\author{
T. HOSOKAWA AND M. YOKOSAWA \\ Department of Physics, Ibaraki University \\ 2-1-1 Bunkyou, Mito 310, Japan
}

Several scales' density fluctuations which exist in the early universe will first gravitationally collapse along one axis and make pancake-like structures. If the collapsed baryonic pancake heats up over $10^{4} \mathrm{~K}$ by shock formation, radiative cooling begins to work and mass accretion toward the central region will advance. Because of this effect, mass fraction of the high density layer becomes large. Densities and widths of the layers will reflect masses of structures (e.g. galaxy) which will be formed after caustics. In this respect, we assumed an Einstein-de Sitter universe dominated by cold dark matter $\left(\Omega_{D M}=0.9\right)$ and investigated the evolutions of fluctuations numerically using one-dimensional hydrodynamic plus $\mathrm{N}$-body codes. We applied a new method for larger fluctuation scales; it is a hybrid method of Eulerian PPM and Zeldovich approximation and it can simulate around the central pancake region with high accuracy.

Simulations were performed for several fluctuation scales $(100 K p c-$ $40 M p c$ ), and their amplitudes were determined by a CDM power spectrum. Fragmental scales were analyzed using thin sheet analysis by energy argument. The results indicate that baryonic matter can create disk-like structures that have characteristic mass scale of $10^{8-9} M_{\odot}$ and disk diameter of $0.1-0.2 M p c$ because of the effect of radiative cooling. On the other hand, fragmental scales of dark matter depend strongly on initial fluctuation scales because of the self-similarity of dark matter. We also found a simple relation at the epoch of fragmentation for both baryonic and dark matter as

$$
\frac{\sigma}{d} \simeq \rho_{\text {crit }},
$$

where $\sigma$ is the surface density and $d$ is the diameter of the fragmental disk. This probably be a necessary condition for disk-like fragmentation on pancake collapse. 